\title{
Accelerometer Profile of Motion of the Pelvic Girdle in Breaststroke Swimming
}

\author{
by \\ Zbigniew Staniak ${ }^{1}$ Krzysztof Buśko², Michał Górski ${ }^{1}$, Anna Pastuszak ${ }^{1}$
}

\begin{abstract}
The aim of the study was to develop a method for measurement and analysis of kinematics of the pelvic girdle movement in breaststroke swimming to support training of technical skills. The measurements were performed in five elite breaststroke swimmers. A recording device for triaxial accelerations and triaxial rotational angular velocity was mounted on the dorsal part of the pelvic girdle of the athlete. The task of the athlete was to swim one length of the $50 \mathrm{~m}$ swimming pool with the intensity similar to competition. From the consecutive cycles of swimming motion, we developed individual mean graphical and numerical profiles of the kinematics of the pelvic girdle movement within the average cycle. Mean basic values of the characteristics were compared with the values documented in the literature obtained by means of the video analysis or using the method of measurement of the velocity of the unwound rope. The comparison revealed that the results in the group studied were very similar to the results obtained using other methods and were typical of elite athletes. Analysis of the motion profiles for the pelvic girdle in breaststroke swimmers indicated that the results obtained reflected individual technique of performing individual phases of swimming cycles. The proposed measurement method, presentation and analysis of the profile of the pelvic girdle motion in breaststroke swimming represents a good tool for fast and effective biomechanical evaluation of motion technique components.
\end{abstract}

Key words: swimming, acceleration, velocity, technique of motion measurement, propulsion.

\section{Introduction}

The dominant role in the research on biomechanics of swimming and in sports practice is played by cinematographic analyses (Craig et al., 2006; Leblanc et al., 2005; Seifert et al., 2005; Strzała et al., 2014). Precise video analyses are performed using high-class video cameras in adapted swimming pools (Harald et al., 2014). However, it should be noticed that the results of video analyses, especially changes in the velocity and accelerations, are substantially smoothed and do not allow for capturing subtle differences in the movement technique. Another limitation is that only a few cycles can be precisely analysed (Dadashi et al., 2013).

Apart from the cinematographic methodologies for measurement of movement velocity, there are also velocity meter systems where velocity measurements are based on the velocity of unwinding the rope attached to the swimmer's belt (Chollet et al., 2004; Craig et al., 2006; Leblanc et al., 2005; Seifert et al., 2005). The absolute value of changes in velocity is typically analysed and the mean acceleration of motion in individual parts of the cycle is calculated (Leblanc et al., 2007). The limitations of this method include, among others, possibilities of performing measurements for only one length of a $25 \mathrm{~m}$ swimming pool, an additional load to the swimmer's body caused by the resistance of the rope and likelihood of collision between the rope and swimmer's limbs (Dadashi et al., 2013).

With miniaturization of accelerometers

1 - Institute of Sport - National Research Institute, Dept. of Biomechanics, Warszawa, Poland.

2 - Dept. of Biomedical Basis of Physical Culture, Kazimierz Wielki University, Bydgoszcz, Poland. 
and gyroscopes, they become more frequently used for motion analysis, also in swimming. Attached to selected segments of the swimmer's body, they allow for analysis of motion kinematics of the wrist (Ohgi et al., 2003), pelvic girdle or body trunk (Davey et al., 2008; Stamm et al., 2013) or many body segments when simultaneously using special suits stabilizing fixation of the sensors (Dadashi et al., 2012; Mooney et al., 2016). Using proper software helps obtain information about the swimmer's movement technique in an easier, faster and cheaper way compared to the cinematographic methods.

From the standpoint of analysis of general movement technique, with particular focus on changes in velocity of motion within a single cycle, analysis of motion kinetics of the centre of mass or the point near the centre of mass seems to be purposeful. Located near the centre of mass, the pelvic girdle is a body segment that transfers mutual effect of lower limbs and upper limbs, with mutual synchronization of activities defining the movement technique (Chollet et al., 2004). From the standpoint of the measurement, dorsal part of the pelvic girdle is an optimal place for fixation of a measurement recording device (Mooney et al., 2016) in a manner that does not disturb individual movement technique, particularly during breaststroke swimming.

The aim of the study was to develop a method for measurement and analysis of kinematics of the pelvic girdle movement in breaststroke swimming to support training of technical skills.

\section{Material and Methods}

\section{Participants}

Five elite male athletes from the Polish National Team of breaststroke swimmers were examined in the study (age: $20.6 \pm 2.30$ years, body height: $186.4 \pm 3.36 \mathrm{~cm}$, body mass: $80.8 \pm$ $2.49 \mathrm{~kg}$ ). The record results of swimmers in breaststroke swimming at the long swimming pool were from 27.24 to $28.62 \mathrm{~s}(50 \mathrm{~m})$ and from 58.68 to $1: 02.36 \mathrm{~min}$ (100 m).

\section{Measures}

An integrated recording device was used for the measurements (REJ006, JD Jarosław Doliński, Poland) with an in-built triaxial accelerometer and a triaxial gyroscope. The device, with the dimensions of $65 \times 50 \times 30 \mathrm{~mm}$ and mass of $150 \mathrm{~g}$, was placed in a light, stiff foam formed in a manner that minimized hydrodynamic resistance and ensured stable fixation on the dorsal portion of the pelvic girdle of the swimmer (Figure 1).

The centre of the recording device was located at the initial height of the sacrum. The recording device with the foam was fixed with a special belt made in half of an elastic band and in half of inextensible rope in a manner that ensured stable fixation and minimization of hydrodynamic resistance. The upper edge of the elastic band was located at the level of 2 to $3 \mathrm{~cm}$ below the iliac crest.

The acceleration components were measured directly: Av - along a vertical axis, Ab - along a transverse axis, As - along a sagittal axis of the swimmer's body and components of angular velocities of rotation: $\mathrm{Gv}$ - around a vertical axis, $\mathrm{Gb}$ - around a transverse axis, Gs - around a sagittal axis of the swimmer's body, actually connected with the system of reference with respect to the casing of the recording device.

The acceleration was measured over the measurement range of $\pm 6 \mathrm{~g}$ using a low-pass antialiasing filter with a cut-off frequency of $292 \mathrm{~Hz}$. The angular velocity of rotation was measured over the measurement range of $\pm 500 \% \mathrm{~s}$ using a low-pass anti-aliasing filter with a cut-off frequency of $93 \mathrm{~Hz}$. The values measured were sampled with a frequency of $400 \mathrm{~Hz}$.

Measurement accuracy for the acceleration components was verified in statistic condition with respect to the gravitational acceleration. The absolute measurement error for acceleration was $\pm 0.2 \mathrm{~m} / \mathrm{s}^{2}$. Measurement accuracy for angular velocities of rotation was verified indirectly based on the measurement and calculation of the angle of rotation of the recording device within the range of 90 degrees around each axis. Absolute error of angle calculations was $\pm 1^{\circ}$. Absolute measurement error for angular velocity of rotation was evaluated as $0.6 \%$ s.

\section{Procedures}

Ethical approval for this study was provided by the Ethics Committee of the Institute of Sport, National Research Institute, Warsaw, Poland. Written informed consent was obtained from participants or their parents if a participant was under 18 years of age. The study was conducted in accordance with the Declaration of Helsinki. 
Measurements were performed during swimming in a $50 \mathrm{~m}$ swimming pool, at submaximal intensity typical of the current training microcycle. The athletes jumped from the starting platform, swimming over one length of the swimming pool using the breaststroke style and performed the flip turn. An individual warmup was performed by athletes before the measurement.

The measurement was repeated three times by one athlete, twice by another one, and performed only once by remaining three swimmers because of the absence of the athletes on the next training national team camps. The measurements were performed in various training microcycles at the beginning of two weeks camps connected with preparation to the nearest competition. A total of eight measurements were used for the analysis.

Based on the directly measured components of accelerations $\mathrm{Av}, \mathrm{Ab}, \mathrm{As}$ and components of angular velocities of rotation $\mathrm{Gv}, \mathrm{Gb}$ and $\mathrm{Gs}$, we calculated the changes in: acceleration $(\mathrm{Ax})$ of translational motion and changes in angular velocity $(\mathrm{Vx})$ of the translational motion in the axis parallel to the water surface, termed acceleration and velocity of translational motion, and vertical acceleration $(\mathrm{Az})$ in the axis perpendicular to the water surface and changes in the angle of inclination of the pelvic girdle with respect to the transverse axis of the swimmer's body.

The profiles measured were smoothed with the low-pass, four-pole Butterworth filter with a cut-off frequency of $20 \mathrm{~Hz}$. The cut-off frequency of the filter was selected using the assumption that the calculated amplitude of changes in the velocity of translational motion should not be suppressed by more than $0.5 \%$ as an effect of filtering of the signals measured and that the collapses of the acceleration profiles, critical from the standpoint of motion analysis, were well noticeable.

Of all individual swimmer's cycles typical of the breaststroke swimming, we determined individual mean graphical characterization of the cycle of profiles of measured and calculated values. The mean numeric profile for the cycle containing arithmetic means of selected values, characteristic points and values measured or calculated, as well as mean values and standard deviations of the relative (with respect to cycle duration) time to reach these values measured from the beginning of the cycle were also calculated. These values are further termed numeric parameters of the profile. The profile obtained was named the AG3DC profile. Calculations were made using the author's software STA1v0 (Zbigniew Staniak, Institute of Sport - National Research Institute, Poland).

\section{Statistical Analysis}

Pearson's correlation coefficients were used to evaluate relationships between the parameters. All computations were performed with STATISTICA software (v. 12.0, StatSoft, USA).

\section{Results}

An example of an individual AG3DC profile for the movement of the pelvic girdle in breaststroke swimming is presented in the diagram in the rectangular coordinate system (Figure 2). The vertical axis of the diagram represents normalized values of profiles. The profiles were standardized with respect to the referential values for the value 1 of each parameter, presented in the left lower corner of the diagram.

The vertical axis of the diagram refers to mean duration of the swimming cycle expressed in percentage terms. The beginning of the cycle was the moment when the profile of the angular velocity of rotation $(\mathrm{Gb})$ of the pelvic girdle around the transverse axis of the swimmer's body changed the sign from negative to positive in the first part of the propulsive activity of the upper limbs. At this point, the angle of pelvic girdle inclination $(\mathrm{KGb})$ had minimal value and started to increase towards the maximal location.

With respect to other local extremes of changes in velocity of translational motion $(\mathrm{Vx})$, the swimming cycle was divided into four characteristic phases according to Craig et al. (2006) and Leblanc et al. (2007).

The first phase of the cycle (upper limb propulsion), denoted with the symbol vAP, started with the beginning of the cycle and ended at the moment of obtaining the first extreme of the velocity of translational motion (maxV $\mathrm{x}_{\mathrm{vAP}}$ ). This phase was connected with the effective upper limb propulsion.

The second phase of the cycle (motion deceleration), denoted as VALR, began at the point of reaching the first local maximum for the 
velocity of translational motion $\left(\operatorname{maxV} \mathrm{x}_{\mathrm{vAP}}\right)$ and ended at the point of reaching the minimum of translational motion velocity (minVx $\left.x_{\mathrm{vALR}}\right)$. It was related with deceleration of the propulsive motion resulting from body trunk resurfacing, forward hand thrust and leg recovery.

The third phase of the cycle (lower limb propulsion), denoted as VLP, started at the moment of reaching the minimum velocity of translational motion (minV $\mathrm{x}_{\mathrm{VALR}}$ ) and ended at the point of reaching the last local maximum velocity of translational motion (maxV $\left.\mathrm{x}_{\mathrm{vLP}}\right)$. This was connected with the effective propulsive activity during leg extension.

The fourth phase of the cycle, denoted as vLAL (gliding), started at the moment of reaching the second local maximum velocity of translational motion $\left(\operatorname{maxVx}_{\mathrm{vLP}}\right)$ and ended with the completion of the cycle. In this phase, a smooth decline in the velocity of translational motion was observed. The decline was maintained by adduction of lower limbs, a gradual decrease in the kinetic energy of the translational motion of the body, insignificant "butterfly" body movements and gravitational falling of the body.

For the purposes of our analysis, the complete cycle was divided into two parts: part 1 (P1) including the first two phases of the cycle (vAP and vALR) and part 2 (P2) that was comprised of the two last phases of the cycle (vLP and vLAL).

Table 1 presents mean value of time to cover one length of the swimming pool and changes in parameters of the profile with regard to translational motion of the pelvic girdle in the swimmers studied.

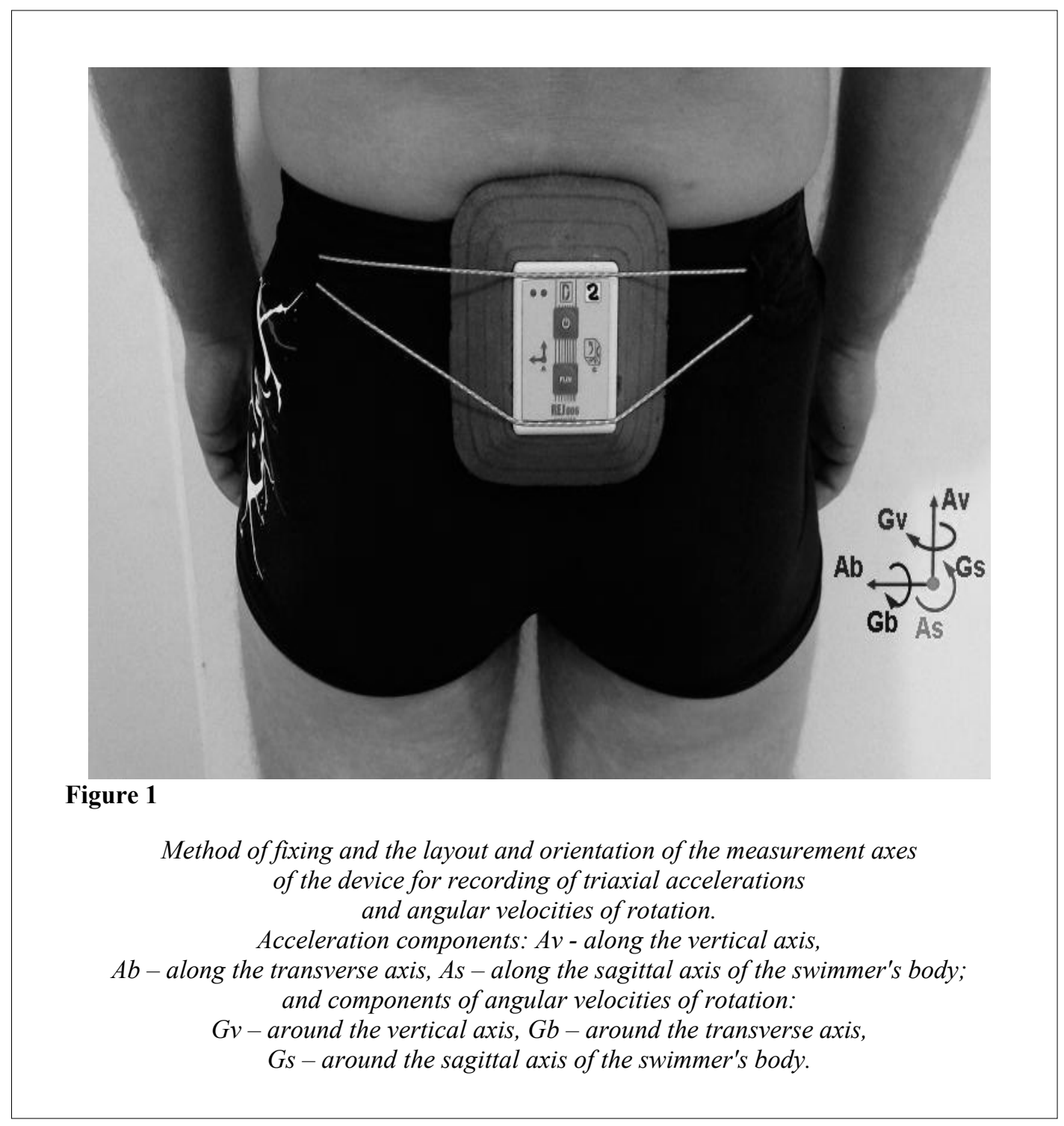




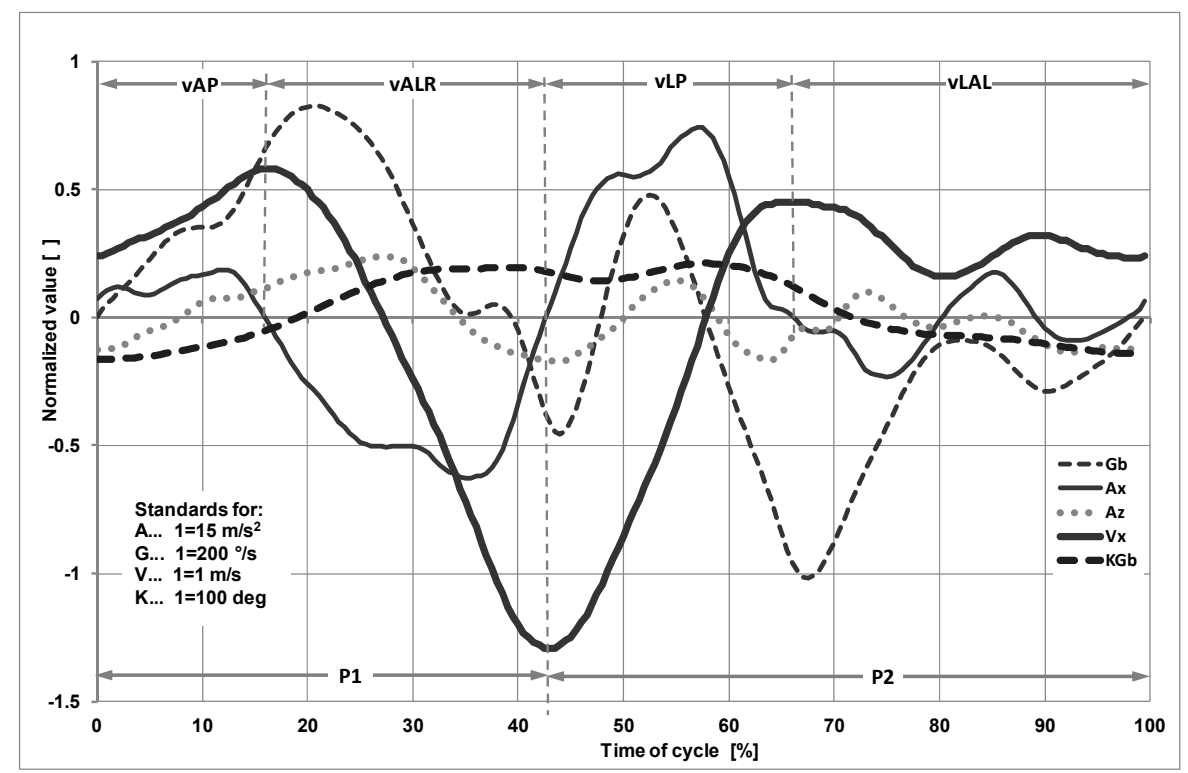

Figure 2

Example of the AG3DC profile of the pelvic girdle motion

for the average cycle of breaststroke swimming

Ax - horizontal acceleration of translational motion,

$A z$-vertical acceleration of translational motion,

$V x$ - change in the velocity of translational motion,

$\mathrm{Gb}$-angular velocity of pelvic girdle inclination around the transverse body axis,

$K G b$-angle of pelvic girdle inclination, vAP - upper limb propulsion,

$v A L R$ - motion deceleration, vLP - lower limb propulsion, vLAL - gliding,

P1 - first part of the cycle, P2 - second part of the cycle.

Table 1

Mean values $( \pm S D)$ of the parameters of the profile describing changes in the velocity of translational motion in the cycle, $n=8$

\begin{tabular}{lllllll}
\hline & $\begin{array}{l}\text { T50 } \\
(\mathrm{s})\end{array}$ & $\begin{array}{l}\mathrm{SR} \\
(\mathrm{c} / \mathrm{min})\end{array}$ & $\begin{array}{l}\text { meanVx } \\
(\mathrm{m} / \mathrm{s})\end{array}$ & $\begin{array}{l}\mathrm{rtmVx} \\
(-)\end{array}$ & $\begin{array}{l}\mathrm{ampVXP1} \\
(\mathrm{m} / \mathrm{s})\end{array}$ & $\begin{array}{l}\mathrm{ampVXP2} \\
(\mathrm{m} / \mathrm{s})\end{array}$ \\
\hline Value & $32.0 \pm 0.8$ & $49.1 \pm 4.6$ & $1.24 \pm 0.13$ & $2.04 \pm 0.38$ & $1.90 \pm 0.12$ & $1.93 \pm 0.20$ \\
\hline
\end{tabular}

T50 - time to swim the $50 \mathrm{~m}$ distance considered as a time between the start

from the platform and the moment of deceleration during the flip turn,

$S R$ - swimming rate expressed by the number of swimming cycles per minute, meanVx-mean value of changes in the velocity of translational motion expressed

in metres per second, $r \operatorname{tm} V x$-dimensionless index of the quotient of the time

at which the velocity $(V x)$ of the propulsive motion is equal or greater than

the mean value of changes in the velocity (meanVx) in the cycle and time at which the value of the velocity of translational motion $(V x)$ is lower than the mean value in the cycle, amp

$V x_{P 1}$ - amplitude of changes in the velocity of translational motion (Vx) in the first part of the cycle (P1), ampV $x_{P 2}$ - amplitude of changes in the velocity (Vx) of the translational motion in the second part of the cycle (P2). 
Table 2

Mean values $( \pm S D)$ of selected parameters of the profile in consecutive characteristic cycle phases, $n=8$

\begin{tabular}{lcccc}
\hline & vAP & vALR & vLP & vLAL \\
\hline RTD $(\%)$ & $19.6 \pm 3.5$ & $23.7 \pm 3.0$ & $23.1 \pm 4.2$ & $33.6 \pm 7.9$ \\
$\operatorname{maxVx}(\mathrm{m} / \mathrm{s})$ & $0.66 \pm 0.11$ & - & $0.68 \pm 0.14$ & - \\
$\operatorname{RTO} \operatorname{maxVx}(\%)$ & $19.6 \pm 3.5$ & - & $66.4 \pm 7.9$ & - \\
$\operatorname{minVx}(\mathrm{m} / \mathrm{s})$ & - & $-1.24 \pm 0.13$ & - & $0.09 \pm 0.10$ \\
$\operatorname{RTOminVx}(\%)$ & - & $43.3 \pm 5.7$ & - & $89.8 \pm 6.3$ \\
$\operatorname{maxAx}\left(\mathrm{m} / \mathrm{s}^{2}\right)$ & $6.2 \pm 1.4$ & - & $20.5 \pm 6.4$ & - \\
$\operatorname{RTO} \operatorname{maxAx}(\%)$ & $12.2 \pm 4.3$ & - & $59.0 \pm 10.0$ & - \\
$\operatorname{minAx}\left(\mathrm{m} / \mathrm{s}^{2}\right)$ & - & $-12.8 \pm 1.9$ & - & $-10.2 \pm 5.2$ \\
$\operatorname{RTO} \operatorname{minAx}(\%)$ & - & $32.2 \pm 4.0$ & - & $68.8 \pm 8.6$ \\
$\mathrm{KGbk}(\mathrm{deg})$ & $0.0 \pm 5.3$ & $17.3 \pm 5.7$ & $11.7 \pm 7.9$ & $-12.7 \pm 7.6$ \\
\hline \multicolumn{4}{c}{$v A P-$ the first phase of the cycle, effective upper limb propulsion, }
\end{tabular}

$v L P$ - the third phase, effective lower limb propulsion, vLAL - the fourth phase, gliding,

$R T D$ - relative duration of the cycle phase, $\max V x$,

minVx - maximal and minimal value of the velocity of translational motion,

RTOmaxVx, RTOminVx - relative time to maximal and minimal

value of the velocity of translational motion, $\max A x$,

$\min A x$ - maximal and minimal value of the acceleration of the translational motion,

RTOmaxAx, RTOminAx - relative time to maximal and minimal value

of the acceleration of translational motion, KGbk - value of the angle of inclination of the pelvic girdle at the end of the cycle phase.

\begin{tabular}{|c|c|c|}
\hline \multicolumn{3}{|c|}{$\begin{array}{l}\text { Mean values }( \pm S D) \text { of selected parameters of the profile } \\
\text { in the first and the second part of the cycle }\end{array}$} \\
\hline $\mathrm{N}=8$ & P1 & P2 \\
\hline $\operatorname{maxAz}\left(\mathrm{m} / \mathrm{s}^{2}\right)$ & $4.0 \pm 1.3$ & $5.4 \pm 3.3$ \\
\hline RTOmaxAz (\%) & $29.5 \pm 4.5$ & $53.7 \pm 4.7$ \\
\hline $\min \mathrm{Az}\left(\mathrm{m} / \mathrm{s}^{2}\right)$ & $-5.1 \pm 1.1$ & $-3.8 \pm 2.3$ \\
\hline RTOminAz (\%) & $42.1 \pm 5.3$ & $60.3 \pm 3.6$ \\
\hline $\operatorname{maxGb}(\operatorname{deg} / \mathrm{s})$ & $154.1 \pm 29.3$ & $136.0 \pm 60.4$ \\
\hline RTOmaxGb(\%) & $25.4 \pm 5.7$ & $57.3 \pm 8.1$ \\
\hline $\min G b(\operatorname{deg} / \mathrm{s})$ & $-133.8 \pm 30.8$ & $-198.7 \pm 50.2$ \\
\hline RTOminGb (\%) & $45.5 \pm 7.2$ & $68.7 \pm 8.0$ \\
\hline \multicolumn{3}{|c|}{$\begin{array}{l}\text { P1 - the first part of the cycle, } P 2 \text { - the second part of the cycle, } \\
\text { aaxAz, minAz-maximal and minimal value of vertical acceleration, } \\
\text { RTOmaxAz, RTOminAz-relative time to maximal } \\
\text { and minimal value of vertical acceleration, max Gb, } \\
\text { maximal and minimal value of angular velocity of pelvic girdle inclination, } \\
\text { RTOmaxGb, RTOmin } G b \text { - relative time to maximal } \\
\text { and minimal value of angular velocity of pelvic girdle inclination. }\end{array}$} \\
\hline
\end{tabular}


Table 2 presents selected mean values $( \pm$ SD) of the parameters in individual phases of the swimming cycle. Local extremes of the values in the translational motion $(\mathrm{Vx})$ may be observed at the end of each of the three first phases of the cycle. The last (fourth) phase (vLAL) contains a local minimum with regard to velocity. Local extremes of acceleration $(\mathrm{Ax})$ of the translational motion occur within each characteristic phase of the cycle.

Characteristic values of the profile regarding the vertical acceleration $(\mathrm{Az})$ and angular velocity of pelvic girdle inclination $(\mathrm{Gb})$ occur within neighbouring characteristic parts of the cycle. The calculated maximal and minimal value of these profiles that occur in the first and the second part of the cycle are presented in Table 3.

Statistically significant Pearson's correlations with the time to cover one length of the swimming pool $(\mathrm{T} 50)$ were found $(\mathrm{p}<0.05)$ for minAxvLAL $(r=-0.76), \operatorname{maxAzP} 2(r=0.84)$ and RTOminAzP2 ( $\mathrm{r}=0.91)$.

\section{Discussion}

The results obtained in the present study were compared to the findings published in the literature concerning changes in the velocity of translational motion of the hip joint axis obtained using video recording and measurement of the velocity of unwinding the rope (termed "velocity meter") attached to the swimmer's belt (Chollet et al., 2004; Leblanc et al., 2007). Generally, the obtained profiles of changes in the velocity of translational motion $(\mathrm{Vx})$ are consistent with the profiles reported in the aforementioned studies. The characteristic subtle local collapses in the profile are connected with e.g. adduction of lower limbs and initiation of the propulsive activity of the upper limbs.

Leblanc et al. (2005) found absolute mean motion velocity of $1.49 \pm 0.13 \mathrm{~m} / \mathrm{s}$ in elite swimmers when swimming with intensity corresponding to their actual competitive times of the $50 \mathrm{~m}$ distance. Leblanc et al. (2007) also observed that the absolute minimal value of the motion velocity in a cycle in advanced athletes was very similar and amounted to $0.26 \mathrm{~m} / \mathrm{s}$; moreover, the extreme velocities connected with propulsive activity of the lower limbs were higher than the extreme velocities connected with the activity of the upper limbs.
The mean value of changes in velocity $(\mathrm{meanVx}=1.24 \mathrm{~m} / \mathrm{s})$ after summation with the minimal absolute velocity of the translational motion $(0.26 \mathrm{~m} / \mathrm{s})$ presented in the study by Leblanc et al. (2007) demonstrated that mean absolute velocity of the translational motion in the group studied was $1.50 \mathrm{~m} / \mathrm{s}$. This value is very similar to the findings presented in a study by Leblanc et al. (2007) concerning mean values in the range of $1.49-1.53 \mathrm{~m} / \mathrm{s}$ and to the mean velocity achieved by the participants in our study when covering the distance of $50 \mathrm{~m}(1.55 \mathrm{~m} / \mathrm{s})$, calculated from the mean time for $50 \mathrm{~m}$ (T50) with the time of start from the platform.

The mean values of the amplitude of changes in the velocity connected with upper limb propulsion $(\mathrm{ampVxP} 1=1.90 \pm 0.12 \mathrm{~m} / \mathrm{s})$ and the correlated lower limb propulsion $(\mathrm{ampVxP} 2=1.93$ $\pm 0.20 \mathrm{~m} / \mathrm{s}$ ) are similar. Numerous studies have used video analysis to determine the duration of individual parts of the cycle in order to establish coordination correlations between activities of upper and lower limbs in breaststroke swimming. Leblanc et al. (2005) compared elite athletes with non-elite ones. Elite athletes had mean velocity of $1.49 \pm 0.13 \mathrm{~m} / \mathrm{s}$ and the swimming rate of $47.8 \pm$ $7.99 \mathrm{cycle} / \mathrm{min}$. These values are similar to the results recorded in our group of swimmers. For this reason analysis of duration of individual cycle phases in our study was compared to the one reported by Leblanc et al. (2005).

In our study, relative mean duration of the first phase of the cycle $(\mathrm{TvAP}=19.6 \pm 3.5 \%)$ reflected by the effective propulsion of upper limbs was nearly twice shorter than duration of propulsive activity of upper limbs (Up limbs prop $=34.38 \pm$ $6.05 \%$ ) documented in a study by Leblanc et al. (2005). This difference was expected due to the fact that a study by Leblanc et al. (2005) evaluated the duration of the propulsive phase based on the range of propulsive motion of the upper limbs and the TvAP index relates to propulsive motion of the upper limbs connected with effective acceleration. Studies by Chollet et al. (2004) and Leblanc et al. (2007) presented the demonstrative profiles of changes in the velocity with marked initial and final points of swimmer's limb activity. The beginning of the range of propulsive motion of the upper limbs was indicated at the moment of obtaining the local minimum of velocity that occurred after the velocity maximum connected 
with lower limb propulsion; the end of the range was found after obtaining the velocity maximum connected with propulsive activity of the upper limbs. In our study, the total duration of the first cycle phase (TvAP) and time from the velocity minimum (minVvLAL) to the end of the cycle accounted for around $30 \%$ of the cycle. Given that the range of the propulsive motion of the upper limbs ends at around 4 to $6 \%$ of cycle duration after reaching the velocity maximum, it should be stressed that the quantitative results of our study determining duration of the propulsive activity of the upper limbs are consistent with the findings of Leblanc et al. (2005).

The relative duration of the second phase of the cycle (TvALR $=23.7 \pm 3.0 \%)$, connected with deceleration of the motion due to leg and arm recovery was insignificantly longer than the relative duration of the leg recovery presented by Leblanc et al. (2005) (Leg.recov. $=26.34 \pm 3.90 \%$ ) and relative duration of arm recovery (Arm.recov. $=27.82 \pm 3.92 \%$ ). However, the difference in mean values was relatively small and, with substantial values of standard deviations of these values, our findings should be considered as similar.

The relative mean duration of the third phase of the cycle (TvLP $=23.1 \pm 4.2 \%$ ) should be consistent with the findings presented by Leblanc et al. (2005) for leg propulsion time (Leg prop. = $18.32 \pm 3.81 \%$ ). The difference in this variable may have been caused by several factors. One of them may be the fact that the end of the leg propulsion phase is not unequivocally specified in the demonstrative figures presented in the report by Leblanc et al. (2005). Furthermore, the propulsive motion of lower limbs is one of the most difficult components of breaststroke swimming technique. As the value of vertical motion (acceleration Az) of the pelvic girdle at around velocity extreme rises substantially, the respective movement of lower limb adduction after extension may be the reason for elongation of the duration of effective lower limb propulsion.

There is no unequivocal reference in the literature for the duration of the fourth phase of the cycle (TvLAL $=33.6 \pm 7.90 \%$ ) since it comprises the beginnings and ends of different ranges of lower limb and upper limb motion. All individual profiles of the athletes studied show characteristic fluctuations of velocity, similar to those presented in the study by Leblanc et al.
(2005), connected with the last phase of leg adduction, actual gliding and the beginning of the range of motion of propulsive activity of the upper limbs. The propulsive activity of leg adduction may be evaluated qualitatively based on the shape and speed of decline in the profile of velocity after reaching the velocity maximum connected with effective propulsion of lower limbs. The effectiveness and smoothness of propulsion in the first part of the propulsive range of motion of upper limbs can be evaluated qualitatively based on the velocity and acceleration of translational motion (Ax) in the last phase of the cycle.

The relatively small standard deviations for the mean change of the velocity of translational motion (meanVx) and amplitudes of changes in velocities connected with propulsive activity of the upper limbs (ampVxP1) and lower limbs (ampVxP2) and their time support the thesis that the group of athletes analysed in our study was in general similar in motor terms.

Analysis of acceleration of the translational motion $(\mathrm{Ax})$ and vertical acceleration $(\mathrm{Az})$ allows for a precise conclusion on the motion smoothness and local changes in motion kinetics. Motion smoothness depends on coordination of activity of body segments that generate propulsion. This is demonstrated by a noticeable decline in the smoothness of translational acceleration $(\mathrm{Ax})$ in the second (vALR) and third (vLP) part of the cycle (Figure 2), which is in general imperceptible in the profile of changes in velocity.

The significant negative correlation found for minimal value of acceleration of translational motion (minAxvLAL) (-0.76, p < 0.05) and significant high correlations observed for maximal value of vertical acceleration (maxAzP2, 0.84, p < 0.05 ) with duration of swimming the $50 \mathrm{~m}$ distance (T50) show that the ability to maintain smooth movements in the last phase of the cycle may have a positive effect on performance in the race.

The results obtained for the amplitude of changes in the velocity of translational motion $(\mathrm{ampVx}, \mathrm{r}=0.64)$ and maximum velocity of lower limb propulsion (maxVxvLP, $r=0.48$ ) show that, according to theoretical expectations, large amplitude of velocity changes was not conducive to obtaining better time in the group of athletes studied. 
The negative correlation $(\mathrm{r}=-0.52)$ found for the velocity minimum of the translational motion (minVxvLAL) obtained in the last part of the cycle connected with completion of the gliding phase shows that the ability to maintain high velocity in this part of the cycle has a positive effect on performance in the race.

The correlation between the swimming rate (SR) with T50 $(\mathrm{r}=-0.50)$ demonstrates that, similarly to other sports based on cyclic movements, the swimming rate is a critical factor in effective breaststroke swimming.

\section{Conclusions}

The profile developed in the study allows for a qualitative and quantitative analysis of synchronization of movements and effective results of propulsive activity of swimmer's limbs in consecutive parts of breaststroke swimming.

The analyses and comparison of the results obtained in the study with the data available in the literature reveal that the suggested method of measurement, presentation and analysis of the AG3DC profile of pelvic girdle motion in breaststroke swimming may offer a good tool for general evaluation of movement technique as well as quick and effective biomechanical assessment of selected components of movement technique in breaststroke swimming.

\section{Acknowledgements}

The study was supported by Ministry of Sport and Tourism (Grant No. 2015.056/40/BP/DWM). The results from this study were presented in the abstract form at the conference: „International Conference of the Polish Society of Biomechanics, Biomechanics 2016, 5-7 September 2016" and published in the book of abstracts.

\section{References}

Chollet D, Seifert L, Leblanc H. Evaluation of Arm-Leg Coordination in Flat Breaststroke. Int J Sports Med, 2004; 25: 486-495

Craig AB, Termin B, Pendergast DR. Simultaneous Recordings of Velocity and Video During Swimming. Portuguese Journal of Sport Sciences, 2006; 6 (suppl. 2.): 32-35

Dadashi F, Crettenand F, Millet GP, Aminian K. Front-Crawl Instantaneous Velocity Estimation Using a Wearable Inertial Measurement Unit. Sensors, 2012; 12(10): 12927-12939

Dadashi F, Millet GP, Aminian K. Inertial measurement unit and biomechanical analysis of swimming. Schweizerische Zeitschrift für Sportmedizin und Sporttraumatologie, 2013; 61(3): 28-33

Davey N, Anderson M, James D. Validation trial of an accelerometer-based sensor platform for swimming. Sports Technology, 2008; 1(4-5): 202-207

Leblanc H, Seifert L, Baudry L, Chollet D. Arm-Leg Coordination in Flat Breaststroke: A Comparative Study Between Elite and Non-Elite Swimmers. Int J Sports Med, 2005; 26: 787-797

Leblanc H, Seifert L, Tourny-Chollet C, Chollet D. Intra-cyclic Distance per Stroke Phase, Velocity Fluctuations and Acceleration Time Ratio of a Breaststroker's Hip: A Comparison between Elite and Nonelite Swimmers at Different Race Paces. Int. J. Sports Med, 2007; 28: 140-147

Mooney R, Corley G, Godfrey A, Quinlan LR, ÓLaighin G. Inertial Sensor Technology for Elite Swimming Performance Analysis: A Systematic Review. Sensors, 2016; 16(18): 1-55. doi:10.3390/s16010018

Ohgi Y, Ichikawa H, Homma M, Miyaji C. Stroke phase discrimination in breaststroke swimming using a triaxial acceleration sensor device. Sports Engineering, 2003; 6: 113-123

Olstad BH, Zinner C, Haakonsen D, Cabri J, Kjendlie PL. A new approach for identifying phases of the breaststroke wave kick and calculation of feet slip using 3D automatic motion cracking. BMS 2014Proceedings, 2014: 195-199 
Seifert L, Chollet D. A new index of flat breaststroke propulsion: A comparison of elite men and women. J Sport Sci, 2005; 23(3): $309-320$

Stamm A, James DA, Thiel DV. Velocity profiling using inertial sensors for freestyle swimming. Sports Engineering, 2013; 16(1): 1-11

Strzała M, Krężałek P, Ostrowski A, Kucia-Czyszczoń K, Stanula A, Tyka AK, Sagalara A. Coordination and propulsion and non-propulsion phasesin 100 meter breaststroke swimming. Acta Bioeng Biomech, 2014; 16 (4): 83-89

Strzała M, Krężałek P, Głab G, Kaca M, Ostrowski A, Stanula A, Tyka AK. Intra-Cyclic Phases of Arm-Leg Movement and Index of Coordination in Relation to Sprint Breaststroke Swimming in Young Swimmers. J Sports Sci \& Med, 2013; 12: 690-697.

\section{Corresponding author:}

\section{Zbigniew Staniak}

Department of Biomechanics, Institute of Sport - National Research Institute,

Trylogii 2/16, 01-982 Warsaw, Poland

Phone: 225699954

E-mail: zbigniew.staniak@insp.waw.pli 